\section{E-040 ALTMETRICS AND NEURO-INTERVENTIONAL SURGERY: WHAT DETERMINES 'TRENDING' IN ONLINE MEDIA?}

N Alotaibi*, P Harker, F Robertson, J Vranic, M Koch, C Stapleton, T Leslie-Mazwi, A Patel. Neurosurgery, Massachusetts General Hospital, BOSTON, MA

10.1136/neurintsurg-2020-SNIS.76

Background Alternative metrics (Altmetrics) are increasingly being used as a new metric of scientific dissemination by most medical journals. The aim of this study is to investigate predictors of higher Altmetric scores within the neuro-interventional surgery scope.

Methods We performed a comprehensive search for neurointerventional related publications using the Altmetric database. Articles were selected based on wide-range search criteria that include terms 'interventional', 'neuroradiology', 'neurosurgery' and 'neuro-intervention'. We studied all online references for any article mentioned on Facebook, Twitter, blogs, and mainstream media sources. The strength of associations between all metrics was studied using Pearson correlation coefficient. Values close to 1 indicates strong correlation.

Results A total of 12,501 articles pertinent to neuro-intervention were obtained from the Altmetric database. These articles had an average of an altimetric score of 8 (95\% CI for the mean 7.7 to 9.2). The strongest correlations for Altmetrics were for the following online mentions: general news outlets (Pearson's r: 0.89), Twitter (0.78), Facebook (0.48) and Reddit (0.45). Interestingly, Wikipedia, peer-reviewed outlets and LinkedIn mentions had the weakest correlations coefficients $(0.11,0.21$, and 0.00 , respectively). Journals with social media
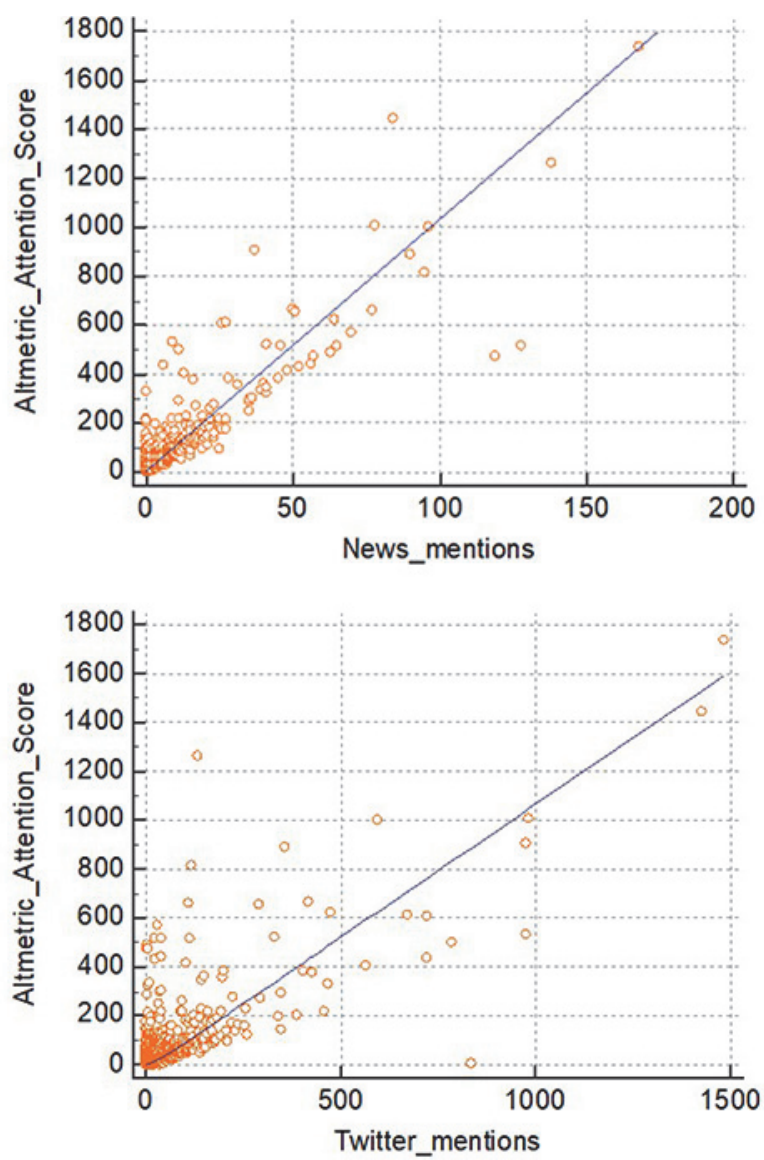

Abstract E-040 Figure 1 presence had the highest average of total altimetric scores compared to journals without social media accounts.

Conclusions Compared to general news outlets and Twitter, professional online networks such as peer-reviewed outlets and LinkedIn did not have a significant association with high altermetric 'trending' scores in neuro-interventional surgery. The long-term impact of these metrics on citations or funding requires a prospective analysis.

Disclosures N. Alotaibi: None. P. Harker: None. F. Robertson: None. J. Vranic: None. M. Koch: None. C. Stapleton: None. T. Leslie-Mazwi: None. A. Patel: None.

\section{E-041 TOWARD ONE-STOP STROKE MANAGEMENT: TIME VARIANT COLOR OVERLAY FOR MULTIPHASE FLAT- PANEL CT ANGIOGRAPHY}

${ }^{1} \mathrm{~K}$ Narsinht, ${ }^{2} \mathrm{~K}$ Mueller, ${ }^{3} \mathrm{M}$ Manhart, ${ }^{1} \mathrm{~S}$ Hetts, ${ }^{1} \mathrm{~T}$ Moore, ${ }^{1} \mathrm{E}$ Chaney, ${ }^{1} \mathrm{D}$ Cooke. ${ }^{1}$ UCSF, San Francisco, CA; ${ }^{2}$ Siemens Healthineers, Mountain View, CA; ${ }^{3}$ Siemens Healthineers, Forchheim, GERMANY

\subsection{6/neurintsurg-2020-SNIS.77}

Introduction Improved functional outcome has been shown in carefully selected patients who are directly transferred to the interventional suite for immediate access to recanalization therapy, bypassing the emergency department (ED) and diagnostic multidetector CT (MDCT). For stroke patients transferred directly to the interventional suite, flat panel CT (FPCT) may provide the necessary diagnostic information regarding intracranial hemorrhage, large vessel patency, clot extent, and collateral supply. However, the large amount of data acquired, typically including 10 phases of contrast-enhancement, can be difficult to review and interpret quickly in the acute stroke setting, and its diagnostic equivalence to MDCT has not been validated. A display format that incorporates all of the multiphasic information into a single time-variant color map may be helpful in facilitating rapid interpretation.

Materials and Methods Between January and October 2019, six acute ischemic stroke patients at a tertiary urban hospital underwent multiphasic FPCT after conventional stroke evaluation with MDCT in the ED. IRB approval was obtained. Data was post-processed on a separate workstation, allowing dynamic visualization of ten $3 \mathrm{D}$ volumes in different phases of contrast enhancement, enabling evaluation for hemorrhage, occlusion site, clot extent, and collateral flow. We retrospectively processed these multiphasic scans in a fully automated workflow including rigid motion correction, orbitomeatal line alignment, mask image subtraction, maximum intensity projection (MIP) generation, and production of a single color-coded MIP volume condensing the data contained in the 10 phases of contrast enhancement.

Results Intracranial hemorrhage was not detected in any case, either on MDCT or FPCT. Color-coded MIP images enabled confident diagnosis of large vessel occlusion, clot extent, and collateral flow. The time to acquire FPCT in IR was faster than that to acquire MDCT in the ED (2 vs $17 \mathrm{~min}$ ). Theoretical time savings averaged $68 \mathrm{~min}$. All patients underwent subsequent mechanical thrombectomy (with TICI 2b/3 results). FPCT perfusion maps were equivalent to MDCT perfusion maps, and accurately predicted core infarct volume on follow-up MRI/CT, as compared by manual segmentation methods.

Conclusion Flat panel CT angiography is a promising tool to enable one-stop shop management of acute ischemic stroke 